\title{
Corela
}

Cognition, représentation, langage

HS-14 | 2013

Calcul du sens et contexte

\section{Calcul du sens et contexte}

Jocelyn Benoist, Gilles Col et Thierry Poibeau

\section{OpenEdition}

Journals

Édition électronique

URL : http://journals.openedition.org/corela/3099

DOI : 10.4000/corela.3099

ISSN : $1638-573 X$

Éditeur

Cercle linguistique du Centre et de I'Ouest - CerLICO

\section{Référence électronique}

Jocelyn Benoist, Gilles Col et Thierry Poibeau, « Calcul du sens et contexte », Corela [En ligne], HS-14 2013, mis en ligne le 25 juin 2014, consulté le 22 septembre 2020. URL : http:// journals.openedition.org/corela/3099; DOI : https://doi.org/10.4000/corela.3099

Ce document a été généré automatiquement le 22 septembre 2020

\section{(c) (i) (2) (2)}

Corela - cognition, représentation, langage est mis à disposition selon les termes de la licence Creative Commons Attribution - Pas d'Utilisation Commerciale - Partage dans les Mêmes Conditions 4.0 International. 


\title{
Calcul du sens et contexte
}

\author{
Jocelyn Benoist, Gilles Col et Thierry Poibeau
}

1 La question du contexte occupe une place centrale pour l'analyse du langage, aussi bien en linguistique, en philosophie qu'au sein des sciences cognitives. En effet, le sens d'une expression linguistique varie fortement d'un contexte à l'autre et d'une situation donnée à une autre. Mais que faut-il entendre exactement par contexte ? Comment le définir ? Le contexte est- il assimilable à la notion de situation? à la notion d'usage? Ce sont quelques-unes des questions qui ont été abordées au sein du groupe de travail sur «la construction du sens en contexte ", mêlant des linguistes et des philosophes de l'Ecole Normale Supérieure de Paris durant l'année 2011/2012. A l'issue de ces discussions, une journée d'étude organisée à l'ENS en juin 2012 s'est proposé d'aborder ces questions avec un ensemble d'orateurs venus d'horizons aussi variés que la philosophie et la linguistique, mais aussi de l'informatique et du traitement automatique des langues. Les communications présentées lors de la journée sont visibles en ligne (http:// www.lattice.cnrs.fr/Le-calcul-du-sens-en-contexte). Le présent volume, quant à lui, prolonge les débats en reprenant deux présentations (Bruno Ambroise et Nick Riemer), auxquelles a été ajoutée une troisième contribution portant sur la différence entre « contextualisme » et « sémantique pragmatique » (Charlotte Gauvry).

Dans l'héritage des travaux inauguraux de Gottlob Frege et du fameux «principe de contexte » de la préface aux Fondements de l'arithmétique, la notion de "contexte » a gagné un surcroît d'intérêt dans la philosophie du langage de la seconde moitié du $\mathrm{XX}^{\mathrm{e}}$ siècle, au prix de grandes confusions. En philosophie du langage, une conception largement répandue veut effectivement que la signification d'une phrase soit analysable en termes d'une proposition qui permet de spécifier les conditions de vérité de la phrase énoncée. La signification permettrait ainsi de déterminer immédiatement dans quelles conditions l'énonciation d'une phrase qui en est dotée est vraie. A partir de ce constat, Bruno Ambroise présente le point de vue de Charles Travis qui s'oppose rigoureusement à cette conception. Travis entend découpler la signification des mots de leurs conditions de vérité pour montrer, au moyen d'une argumentation contextualiste, que la vérité n'est pas une propriété sémantique des énoncés, mais bien plutôt une dimension pragmatique d'évaluation des énoncés, qui se fait en contexte. Ce 
faisant, il ne conteste pas tant la position frégéenne qu'il n'en reprend certains éléments pour les associer aux idées anti-platonistes de J.L. Austin.

L'article de Charlotte Gauvry tente de contribuer à la clarification des confusions sur la notion de "contexte » en proposant une distinction entre le "contextualisme ", tel qu'il se définit dans un héritage wittgensteinien et austinien, et la "sémantique pragmatique ». Il espère ainsi montrer que la sémantique pragmatique dont l'échelle d'analyse est la proposition, tout en considérant certains éléments de contexte extrasémantique, en reste à l'idée que l'énoncé a un contenu propositionnel susceptible d'être évalué en termes de vérité et de fausseté et que le sens de l'énoncé est déterminé par des "conditions de vérité » (à savoir la manière dont le monde devrait être pour que l'énoncé soit vrai). La sémantique pragmatique en reste ainsi à une analyse sémantique de la phrase. A contrario, Charlotte Gauvry défend l'idée que le «contextualisme radical» bien compris refuse précisément de s'en tenir à cette approche sémantique de l'énoncé et définit le contexte non pas comme un élément de « contenu » de la phrase mais comme la norme qui détermine un acte de langage en tant qu'acte.

4 Après deux illustrations du rôle du contexte, l'article de Nick Riemer aborde plutôt deux effets de contexte sur la représentation du contenu sémantique, en considérant les implications que peuvent avoir ces effets pour la modélisation sémantique. Le premier effet est celui de la sous-minimalité sémantique où le contexte peut rendre le contenu sémantique minimal (définitionnel) redondant pour le traitement cognitive réussi (la compréhension) d'une expression. Le second effet concerne la manière dont le contexte de discours influe sur la nature des représentations sémantiques en-ligne. L'article établit ainsi une différence entre le discours planifié et le discours libre définis comme deux contextes différents pour ce qui est de la nature des représentations sémantiques sous-jacentes. En discutant de ces deux effets, Nick Riemer remet en question le statut empirique des représentations dont on fait l'hypothèse habituellement en sémantique linguistique, et augmente le répertoire des intéractions sens-contexte dont il faut rendre compte dans une théorie naturaliste de la compétence sémantique.

\section{AUTEURS}

\section{JOCELYN BENOIST}

Université Paris 1/ Archives Husserl

\section{GILLES COL}

Université de Poitiers/ FoReLL/ LaTTiCe

\section{THIERRY POIBEAU}

CNRS/ LaTTiCe 